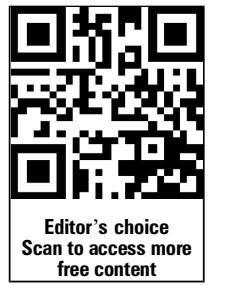
published online only. To view please visit the journal online (http://dx.doi.org/10.1136/ heartjnl-2013-303926).

${ }^{1}$ Department of Radiology and Imaging Sciences, National Institutes of Health Clinical Center, Bethesda, Maryland, USA

${ }^{2}$ Department of Medicine, Johns Hopkins School of Medicine, Baltimore, Maryland, USA

${ }^{3}$ Department of Epidemiology, Johns Hopkins Bloomberg School of Public Health, Baltimore, Maryland, USA ${ }^{4}$ Department of Radiology, Johns Hopkins School of Medicine, Baltimore, Maryland, USA

\section{Correspondence to Dr Joao A C Lima, Department of Medicine, Division of Cardiology, The Johns Hopkins Hospital, 600 N. Wolfe Street, Blalock 524, Baltimore, MD 21287, USA; jlima@jhmi.edu}

Received 7 March 2013 Revised 25 June 2013 Accepted 27 June 2013 Published Online First 19 July 2013

\section{SLinked}

- http://dx.doi.org/10.1136/ heartjnl-2013-304545

To cite: Sibley $\mathrm{CT}_{\text {, }}$ Vavere AL, Gottlieb I, et al. Heart 2013;99:1675-1680.

\title{
MRI-measured regression of carotid atherosclerosis induced by statins with and without niacin in a randomised controlled trial: the NIA plaque study
}

\author{
Christopher T Sibley, ${ }_{1}^{1,2}$ Andrea L Vavere, ${ }^{2}$ Ilan Gottlieb, ${ }^{2}$ Christopher Cox ${ }_{1}^{3}$ \\ Matthew Matheson, ${ }^{3}$ Amy Spooner, ${ }^{2}$ Gustavo Godoy, ${ }^{2}$ Veronica Fernandes, ${ }^{2}$ \\ Bruce A Wasserman, ${ }^{4}$ David A Bluemke, ${ }^{1,4}$ Joao A C Lima ${ }^{2,4}$
}

\begin{abstract}
Objective To evaluate the benefit of niacin in addition to statin therapy on plaque regression among older individuals with established atherosclerosis.

Design Randomised, controlled, double-blind clinical trial.

Setting University outpatient center.

Patients 145 patients older than 65 years, half of them older than 75 years of age, with established atherosclerosis were enrolled.

Interventions Participants received either extended release niacin (1500 mg daily) or placebo in addition to statin therapy to reach their National Cholesterol Education Program-defined low density lipoprotein (LDL) cholesterol target.
\end{abstract}

Main Outcome Measures The primary endpoint was reduction in the wall volume of the internal carotid artery (ICA) measured by MRI.

Results After 18 months, high density lipoprotein cholesterol was higher with statins plus niacin compared with statins alone $(1.6 \pm 0.4$ vs $1.4 \pm 0.4 \mathrm{mmol} / \mathrm{L}$ $p<0.001)$. Both groups had significant decreases in the main outcome measure of ICA wall volume, which regressed at $0.5 \%$ /month (SEM $0.2, p=0.004$ ) in the statins plus placebo group and at $0.7 \% /$ month in the statins plus niacin group (SEM $0.2, p<0.001$ ). There was no difference in the rate of regression between groups ( $p=0.49)$.

Conclusions Treatment with statin therapy to presently recommended LDL levels, with or without niacin, resulted in significant atherosclerosis reduction.

\section{INTRODUCTION}

Patients with previous cardiovascular events are at high risk for recurrent events. Improving risk reduction for older adults is of particular importance, as more than $80 \%$ of cardiovascular deaths occur in those over 65 years of age. ${ }^{1}$ Current guidelines centre on reduction of low density lipoprotein (LDL) cholesterol as the cornerstone of risk reduction. ${ }^{2}$ Despite the impact of statin therapy, significant residual risk remains.

High density lipoprotein (HDL) cholesterol and therapies that raise it have been a focus of attempts to close this gap. There is a clear epidemiologic association between higher levels of HDL and a reduction in cardiovascular events. ${ }^{3}$ The inverse relationship between HDL and cardiovascular events holds true for individuals with above-average levels of HDL, and with advancing age, ${ }^{4}$ though the causality of this relationship is uncertain. Some genetic variants resulting in elevated HDL levels confer no evident protection against myocardial infarction. ${ }^{5}$ While no available drug has effects limited to HDL, a number of medical therapies increase HDL levels in addition to other lipid effects, of which niacin treatment is the longest studied. In the era prior to statins, niacin treatment resulted in regression of angiographically demonstrated atherosclerosis and improved mortality. ${ }^{6} 7$ Combined with statins, niacin has slowed progression, ${ }^{8}$ or induced regression, of atherosclerosis. ${ }^{9-11}$ However, combined statin therapy in these studies was not of the same intensity as recommended by current guidelines. Perhaps most importantly, two large event-driven trials of combination lipid therapy, including niacin and statin therapy, have recently failed to demonstrate incremental benefit with niacin. ${ }^{12} 13$

While a consistently beneficial effect of statins is seen at all levels of LDL, ${ }^{14}$ the benefit of raising HDL with medical therapy remains controversial. This gap in evidence to guide therapy is particularly important in older individuals who are more sensitive to the detrimental effects of polypharmacotherapy, and less well represented in clinical trials. 15

To measure the additional benefit of niacin above statin therapy on plaque regression among older individuals with established atherosclerosis, we conducted a double-blind, randomised, placebocontrolled trial comparing plaque regression induced by the combination of niacin plus statin therapy with that of placebo plus statin therapy alone. We employed MRI to quantify plaque regression in the carotid arteries serially at 6,12 and 18 months. We hypothesised that patients treated with niacin would have a decrease in carotid atherosclerosis compared with statin therapy alone. The primary outcome of this trial was change in internal carotid artery (ICA) wall volume over 18 months.

\section{METHODS}

Study design

The National Institute on Aging Plaque Study (NIA Plaque) was an investigator-initiated randomised, 
placebo-controlled, double-blind trial. The protocol was approved by the Johns Hopkins Institutional Review Board, and all patients provided written informed consent (clinicatrials.gov NCT00127218).

Participants were over age 65 years with a history of cardiovascular events or evidence of atherosclerosis at angiography $(>50 \%$ stenosis) or non-invasive imaging, with baseline $\mathrm{LDL}<3.24 \mathrm{mmol} / \mathrm{L}$ if already on statin therapy and $<3.89 \mathrm{mmol} / \mathrm{L}$ if untreated. There were no restrictions on HDL level. Ineligibility criteria were current use or intolerance of niacin, contraindication to MRI or gadolinium contrast, liver dysfunction, or significant renal failure (GFR $<60 \mathrm{~mL} / \mathrm{min} /$ $\left.1.73 \mathrm{~m}^{2}\right)$.

Participants were randomised in a $1: 1$ ratio to receive niacin or placebo, using a random number schema stratified to ensure equal numbers between age 65-74 years and 75 years and above. Extended release niacin (Abbott, Abbott Park, IL), or corresponding placebo, was titrated to a target dose of $1500 \mathrm{mg}$ daily over 12 weeks. All participants received investigatoradministered statin therapy to achieve LDL goals as specified by current guidelines. ${ }^{16}$ A minimum pill count compliance of $80 \%$ was required to maintain enrolment.

The primary outcome measure was change in ICA wall volume over 18 months (figure 1). Secondary outcomes were HDL, LDL, volumes of ICA lumen, ICA lipid core, common carotid artery (CCA) wall, CCA lumen and CCA lipid core. Carotid MRI was performed at baseline, 6, 12 and 18 months of follow-up.

Participants and investigators were blinded to treatment group assignments. Investigators responsible for titration of therapy and patient follow-up were unaware of imaging, and imaging analysts were blinded to all clinical data, such as complaint of flushing or HDL levels, which may otherwise compromise blinding. The trial was monitored by an independent data and safety monitoring board. The study ended after target enrolment was reached and patients completed the planned 18 months of follow-up.

\section{MRI protocol}

The carotid MRI techniques employed have been previously described in detail ${ }^{17}$ and successfully deployed in a large, multicentre cohort study. ${ }^{18}$ MRI was performed using a $1.5 \mathrm{~T}$ scanner (CVI, General Electric Medical Systems, or Avanto, Siemens Medical Solutions) and carotid coils (Machnet). T1 and T2 weighted, fat suppressed, black blood images were obtained using ECG gating. Postcontrast T1 images were obtained 5 min after an

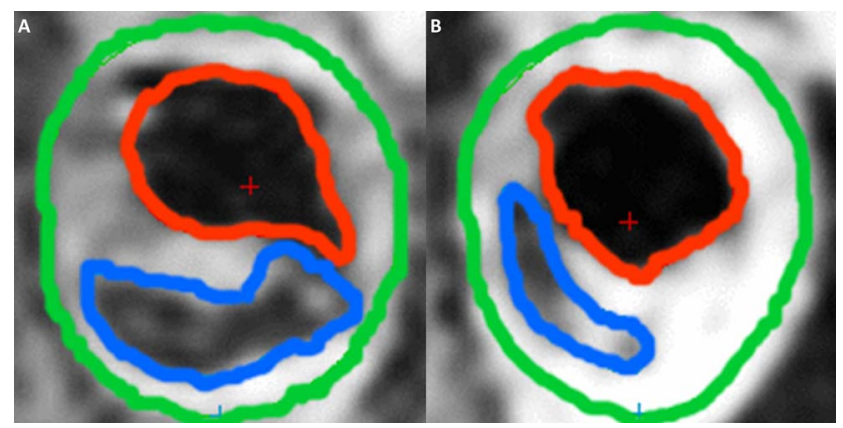

Figure 1 Axial T2-weighted MR image of an internal carotid artery. Green denotes vessel area. Red denotes lumen area. Blue denotes lipid core area. Areas were multiplied by slice thickness and summed by number of slices to calculate volume. Panels depict the same lesion at baseline (A) and 18 months (B). Access the article online to view this figure in colour. intravenous dose of $0.1 \mathrm{mmol} / \mathrm{kg}$ gadodiamide (Omniscan, GE Healthcare). Scan resolution was $0.54 \mathrm{~mm} \times 0.54 \mathrm{~mm} \times 2.0 \mathrm{~mm}$, with five consecutive slices and no gap obtained in each ICA, and one slice obtained in each CCA. Images were positioned at the area of greatest stenosis on scout images. Baseline images were used at follow-up scans to ensure targeting the same segments of artery.

\section{Image analysis}

Measurements were performed using semiautomated software (VesselMass, Leiden University Medical Center) by a single, experienced, blinded observer. These methods have previously been described. ${ }^{19}$ We measured the total area within the external boundary of the vessel, the lumen area, and, when present, the area of lipid core (figure 1). The corresponding volume for each parameter was obtained by multiplying the area in each image by the slice thickness and summing the total number of slices obtained. Wall volume was calculated by subtracting lumen volume from total vessel volume on T2 weighted images. Postcontrast T1 weighted images were used for lipid core delineation.

\section{Statistical methods}

Power calculation assumptions were based on data from a pilot study of high versus standard dose statin therapy on MRI-measured atherosclerosis. ${ }^{20}$ A 12 -month study using comparable methods reported an $8 \%$ decrease in vessel wall volume in statin-treated patients. ${ }^{21}$ Sample size calculations were based on a $15 \%$ difference in plaque volume between study arms calculated by a two-sample t test.

Using a conservative estimated SD of change $40 \%$ larger than that observed in the pilot study, and assuming 50 subjects in each group, the trial had $80 \%$ power to detect a difference in plaque volume with an $\alpha$ of 0.05 . We assumed an attrition rate of 0.30 over 18 months, and accordingly recruited 145 participants to guarantee adequate power with at least 100 patients completing 18 months.

The primary analysis used a mixed model incorporating time as a continuous variable, treatment, and their interaction on ICA wall volume. Analysis included an examination of residuals to check required assumptions of normally distributed errors with constant variance and, where necessary, values were logtransformed to stabilise the variance. For log-transformed outcomes, regression coefficients (slopes) for time are reported as percent change per month. Normally distributed values are reported as means with SD. Non-normally distributed variables are reported as medians and IQRs, and $\chi^{2}$ tests were used for categorical values. Analyses were performed using SAS V.9.2 software. Seven participants who discontinued study drug but continued to undergo imaging follow-up were analysed according to their original treatment group assignment.

\section{RESULTS}

\section{Patient characteristics}

One hundred and forty-five patients were included in the baseline analysis, with 72 randomised to niacin and 73 to placebo. Nineteen discontinued participation during dose adjustment prior to the 6 months, and nine withdrew before 18 months (see online supplementary figure S1). Of 117 participants included in the final endpoint analysis, five in the niacin arm and two receiving placebo discontinued the study drug but continued imaging follow-up according to protocol. 
The participants were older (median 73, IQR 69-77 yrs), and predominantly men $(81 \%)$ with a high prevalence of cardiovascular diseases (table 1).

At baseline, the mean LDL was $2.4 \mathrm{mmol} / \mathrm{L}( \pm 0.8 \mathrm{mmol} / \mathrm{L})$ in the placebo arm and $2.3 \mathrm{mmol} / \mathrm{L}( \pm 0.7 \mathrm{mmol} / \mathrm{L})$ in the niacin arm. There were no significant baseline differences in clinical characteristics $(p>0.05$ for all).

\section{Lipid-lowering therapy}

All participants were on statin therapy at randomisation. Ninety-seven percent had been taking statin drugs for more than 12 months. Most (54\%) were receiving atorvastatin, and the remainder were being treated with rosuvastatin (14\%), simvastatin (13\%), pravastatin (11\%), fluvastatin (6\%) and lovastatin $(2 \%)$. At entry, the mean atorvastatin bioequivalent dose was $16.4 \mathrm{mg}$ in the placebo group and $19.1 \mathrm{mg}$ in the niacin group $(p=0.31)$. There was a significant but clinically small increase in statin dose over 18 months in the statin-placebo group of $4.2 \mathrm{mg}(95 \%$ CI 1.5 to $6.6 \mathrm{mg}, \mathrm{p}=0.003)$, while there was no significant change in statin dose in the niacin group $(1.4 \mathrm{mg}$, 95\% CI 1.0 to $4.5 \mathrm{mg}, \mathrm{p}=0.36$ ). There was no difference in statin dose at study end (statin-placebo $20.6 \mathrm{mg}$, vs statin-niacin $20.5 \mathrm{mg}, \mathrm{p}=0.31$ ). Eighty-eight percent of participants randomised to niacin reached the target $1500 \mathrm{mg}$ dose.

\section{Serum lipids}

Table 2 and figure 2 summarise lipid values at baseline and 18 months. No significant differences existed at baseline. At

Table 1 Demographics and baseline characteristics of the study population

\begin{tabular}{lccc}
\hline & $\begin{array}{l}\text { Placebo } \\
\mathrm{n}=73\end{array}$ & $\begin{array}{l}\text { Niacin } \\
\mathrm{n}=72\end{array}$ & $\mathrm{p}$ Value \\
\hline Age in years & $72(69,77)$ & $73(69,77)$ & 0.64 \\
Sex & & & \\
$\quad$ Male (\%) & $60(82)$ & $58(81)$ & 0.83 \\
$\quad$ Female (\%) & $13(18)$ & $14(19)$ & \\
Race & & & \\
$\quad$ Caucasian (\%) & $65(89)$ & $67(93)$ & 0.74 \\
$\quad$ African-American (\%) & $6(8)$ & $3(4)$ & \\
$\quad$ Other (\%) & $2(3)$ & $2(3)$ & \\
Smoking (ever) (\%) & $30(42)$ & $27(38)$ & 0.73 \\
Diabetes (\%) & $16(22)$ & $11(15)$ & 0.39 \\
HbA1C \% & $6(5.8,6.4)$ & $5.8(5.6,6.3)$ & 0.09 \\
Hypertension (\%) & $53(73)$ & $56(78)$ & 0.57 \\
Prior MI (\%) & $24(33)$ & $22(31)$ & 0.86 \\
Angina (\%) & $37(51)$ & $30(42)$ & 0.32 \\
CABG (\%) & $29(41)$ & $21(31)$ & 0.29 \\
PCI (\%) & $36(50)$ & $32(47)$ & 0.74 \\
PVD (\%) & $8(11)$ & $7(10)$ & $>0.99$ \\
Stroke (\%) & $8(11)$ & $13(18)$ & 0.25 \\
Blood pressure & & & \\
$\quad$ Systolic mm Hg & $134(122,146)$ & $128(118,140)$ & 0.06 \\
$\quad$ Diastolic mm Hg & $70(64,80)$ & $72(64,80)$ & 0.72 \\
Body Mass Index kg/m² & $28(26,35)$ & $28(26,33)$ & 0.41 \\
Abdominal circumference cm & $97(91,104)$ & $97(89,104)$ & 0.86 \\
Serum creatinine mmol/L & $76(61,84)$ & $72(61,84)$ & 0.81 \\
\hline Val in par & & \\
\hline
\end{tabular}

Values in parentheses represent IQR or percentages, as appropriate. Differences tested using Wilcoxon rank-sum (continuous) or Fisher's exact (categorical) tests. MI denotes myocardial infarction. CABG denotes coronary artery bypass grafting. PVD denotes peripheral vascular disease.
18 months, mean HDL in the statin-placebo arm declined from 1.5 to $1.4 \mathrm{mmol} / \mathrm{L}$, while it increased from 1.5 to $1.6 \mathrm{mmol} / \mathrm{L}$ with statin-niacin. The net effect of the addition of niacin to statin therapy was a $17 \%$ relative increase in $\operatorname{HDL}(p<0.001$ for the difference between groups, figure 2).

The majority of patients in both groups reached an LDL of less than $2.6 \mathrm{mmol} / \mathrm{L}$ at 18 months $(85 \%$ placebo, $91 \%$ niacin, $\mathrm{p}=0.39$ ). More patients in the statin-niacin group reached an LDL level under $1.8 \mathrm{mmol} / \mathrm{L} \quad(33 \%$ placebo, 54\% niacin, $\mathrm{p}=0.04)$. LDL declined from 2.4 to $2.0 \mathrm{mmol} / \mathrm{L}$ in the statinplacebo arm $(p<0.001)$ and 2.3 to $1.8 \mathrm{mmol} / \mathrm{L}$ in the statinniacin arm $(p<0.001)$. There was $10 \%$ relative decline in LDL with niacin $(p=0.14$ between groups, figure 3$)$. Triglycerides decreased from 1.5 to $1.3 \mathrm{mmol} / \mathrm{L}$ in the statin-placebo arm, and from 1.5 to $1.0 \mathrm{mmol} / \mathrm{L}$ with niacin $(\mathrm{p}=0.03$ for difference in change between groups). Non-HDL cholesterol declined in both arms and was significantly reduced in statin-niacin patients at 18 months $(2.6 \pm 0.6$ placebo vs $2.3 \pm 0.6 \mathrm{mmol} / \mathrm{L}$ niacin, $\mathrm{p}=0.047)$.

\section{Hypertension and hyperglycemia}

Systolic blood pressure was slightly, though non-significantly, higher at baseline in the placebo arm (median 134 vs $128 \mathrm{~mm} \mathrm{Hg}, \mathrm{p}=0.06$ ). Diastolic blood pressure was similar in both groups at entry (median 70 vs $72, p=0.72$ ). There was no significant difference in the rate of change of systolic $(-0.44 /$ month vs $-0.17 ; \mathrm{p}=0.14)$ or diastolic $(-0.07 /$ month vs -0.18 ; $\mathrm{p}=0.32$ ) blood pressure between groups.

The prevalence of diabetes was similar in both arms $(22 \%$ placebo, $15 \%$ niacin, $\mathrm{p}=0.39)$. There was no difference in haemoglobin A1c at baseline (6.0 placebo, 5.8 niacin; $\mathrm{p}=0.09$ ), and no difference in the rates of change $(-0.005$ vs $0 ; p=0.32)$ between groups.

\section{MRI plaque regression}

There were no baseline differences in wall volume, lumen volume, or lipid content between groups (table 3 and figure 3 ). At baseline, 57\% of patients in the statin-placebo group had a measurable lipid core in the ICA, compared with $51 \%$ in the statin-niacin group. The volume of ICA lipid core was identical in the two groups at randomisation.

Table 3 summarises the outcome measures evaluated by carotid MRI. ICA wall volume declined significantly and to a similar extent in both study arms over the 18 months of follow-up (figure 3). The slope of change in the ICA wall volume was $-0.5 \% /$ month in the statin-placebo group (SEM $0.2 \%, \mathrm{p}=0.004)$ and $-0.7 \%$ month in the statin-niacin group (SEM 0.2\%; $\mathrm{p}<0.001$ ). The change in ICA volume between groups was not significant $(\mathrm{p}=0.49)$. There were no changes in the lumen or lipid core volume of the ICA. The niacin group showed a decrease in the secondary endpoint of CCA wall volume $(-0.4 \% /$ month, $\mathrm{p}=0.03)$, but the difference between the two groups was non-significant $(p=0.26)$.

\section{Safety and events}

Seven participants required revascularisation (three coronary artery bypass grafting, three percutaneous coronary interventions, one carotid endarterectomy), of which five were in the statin-niacin arm. One patient receiving niacin suffered a stroke and one patient receiving statin-placebo died suddenly. Clinical adverse events were low in frequency, and the between-group statistical significance of differences in event rates was not assessed. 
Table 2 Summary of lipid subfraction values

\begin{tabular}{|c|c|c|c|c|c|}
\hline & Baseline value & 18 month value & Slope (\%/month) & $\mathrm{p}$ Value (within group) & p Value (between groups) \\
\hline \multicolumn{6}{|c|}{ Total cholesterol (mmol/L) } \\
\hline Statins+placebo & $4.5 \pm 1.1$ & $4.0 \pm 0.7$ & $-0.6 \%(0.1)$ & $<0.001$ & 0.84 \\
\hline Statins+niacin & $4.4 \pm 0.9$ & $3.9 \pm 0.7$ & $-0.6 \%(0.1)$ & $<0.001$ & \\
\hline \multicolumn{6}{|l|}{$\mathrm{LDL}(\mathrm{mmol} / \mathrm{L})$} \\
\hline Statins+placebo & $2.4 \pm 0.8$ & $2.0 \pm 0.5$ & $-0.8 \%(0.2)$ & $<0.001$ & 0.14 \\
\hline Statins+niacin & $2.3 \pm 0.7$ & $1.8 \pm 0.5$ & $-1.1 \%(0.2)$ & $<0.001$ & \\
\hline \multicolumn{6}{|l|}{$\mathrm{HDL}(\mathrm{mmol} / \mathrm{L})$} \\
\hline Statins+placebo & $1.5 \pm 0.4$ & $1.4 \pm 0.4$ & $-0.3 \%(0.1)$ & 0.007 & $<0.001$ \\
\hline Statins+niacin & $1.5 \pm 0.4$ & $1.6 \pm 0.4$ & $0.4 \%(0.1)$ & $<0.001$ & \\
\hline \multicolumn{6}{|c|}{ Triglycerides (mmol/L) } \\
\hline Statins+placebo & $1.5 \pm 0.9$ & $1.3 \pm 0.6$ & $-0.4 \%(0.3)$ & 0.10 & 0.03 \\
\hline Statins+niacin & $1.5 \pm 0.9$ & $1.0 \pm 0.4$ & $-1.3 \%(0.3)$ & $<0.001$ & \\
\hline \multicolumn{6}{|l|}{ non-HDL (mmol/L) } \\
\hline Statins+placebo & $3.1 \pm 1.1$ & $2.6 \pm 0.6$ & $-0.7 \%(0.2)$ & $<0.001$ & 0.047 \\
\hline Statins+niacin & $2.9 \pm 0.9$ & $2.3 \pm 0.6$ & $-1.2 \%(0.2)$ & $<0.001$ & \\
\hline \multicolumn{6}{|l|}{ TC/HDL ratio } \\
\hline Statins+placebo & $3.3 \pm 1.2$ & $3.0 \pm 0.7$ & $-0.3 \%(0.1)$ & 0.02 & $<0.001$ \\
\hline Statins+niacin & $3.2 \pm 0.9$ & $2.5 \pm 0.5$ & $-1.0 \%(0.1)$ & $<0.001$ & \\
\hline
\end{tabular}

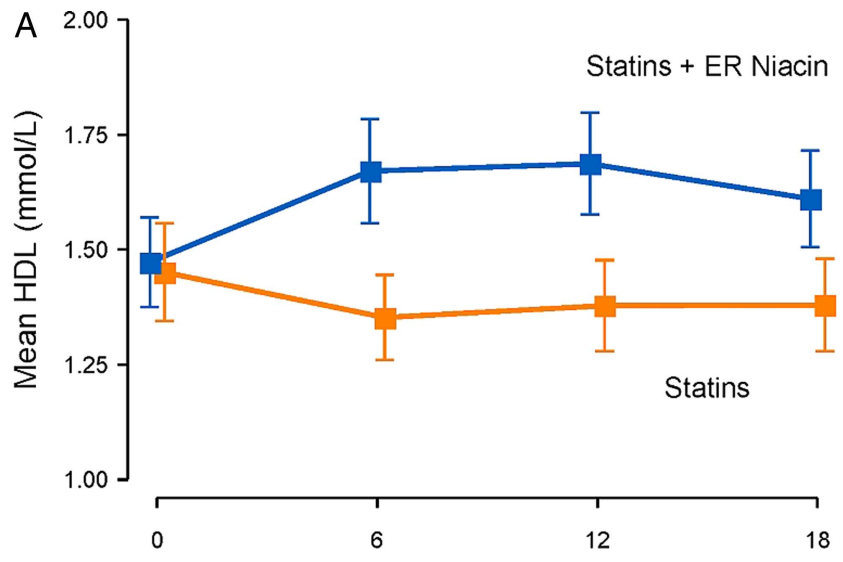

Months

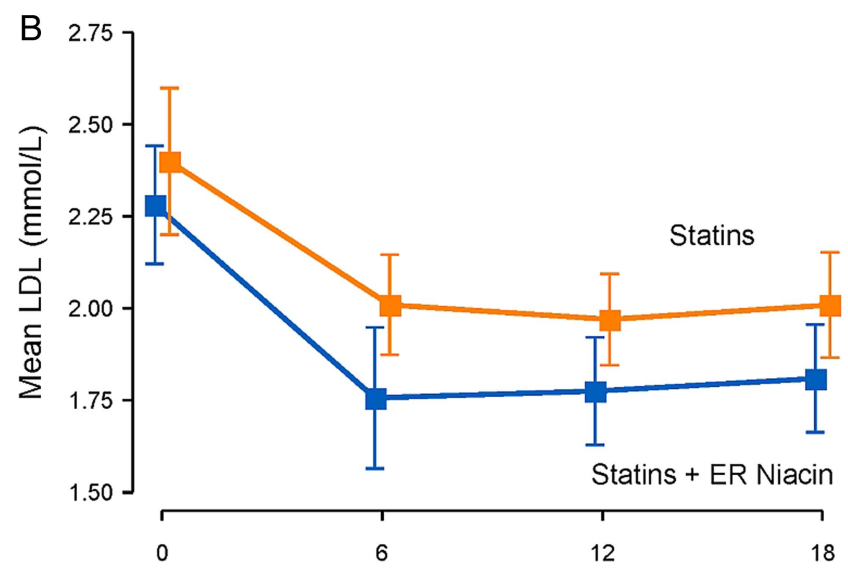

Months

Figure 2 Changes in median levels of LDL and HDL cholesterol in patients receiving statins alone (orange) or in combination with niacin (blue). Vertical bars denote SE. HDL indicates high density lipoprotein. $\mathrm{LDL}$ denotes low density lipoprotein. Access the article online to view this figure in colour.

\section{DISCUSSION}

Treatment with statins to currently recommended levels of LDL, whether alone or in combination with niacin, resulted in significant and sustained reduction in carotid atherosclerosis. Niacin treatment resulted in HDL that was 17\% higher than with statins alone, accounted for, in part, by a significant HDL decline with placebo. Placebo-treated patients required a significant, small, increase in statin dose over 18 months $(4.2 \mathrm{mg}$ atorvastatin equivalent) to reach therapeutic goals, though there was no difference in final statin dose. Both therapeutic strategies resulted in regression of carotid atherosclerosis to a similar degree.

The bulk of existing evidence supporting the use of niacin in the secondary prevention of cardiovascular events derives from studies using imaging measures as their endpoints. Previous reports have extensively explored the differences in patient populations and outcomes in these trials. ${ }^{22}$ An understanding of

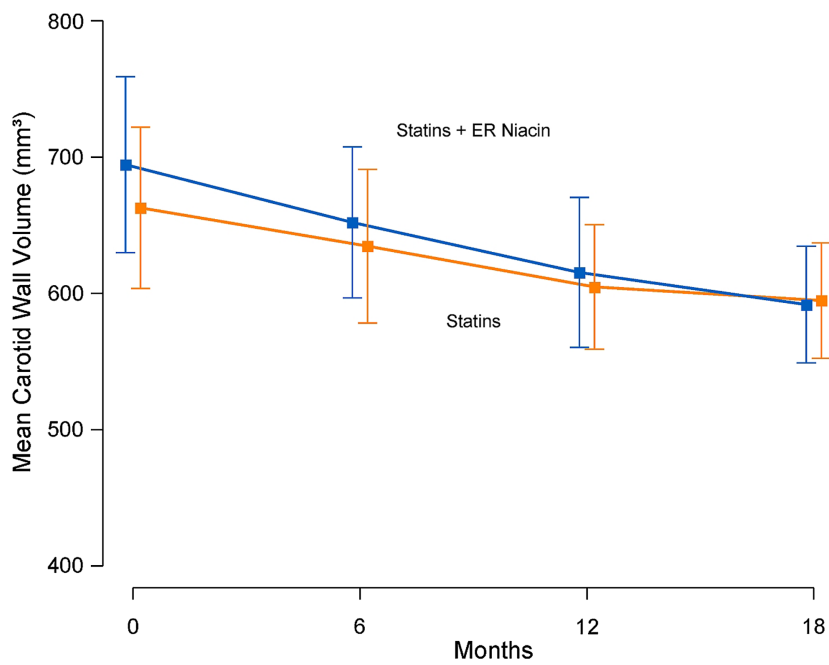

Figure 3 Change in median internal carotid wall volume $\left(\mathrm{mm}^{3}\right)$ over 18 months. Orange denotes statin+placebo. Blue denotes statin+ER niacin. Vertical bars denote SE. Access the article online to view this figure in colour. 
Table 3 Summary of imaging endpoints

\begin{tabular}{|c|c|c|c|c|c|}
\hline & Baseline volume $\mathrm{mm}^{3}$ & 18 month volume $\mathrm{mm}^{3}$ & Slope $\mathrm{mm}^{3} /$ month or $\% /$ month (\%) & p Value (within group) & p Value (between groups) \\
\hline \multicolumn{6}{|l|}{ ICA wall volume* } \\
\hline Statins+placebo & $660 \pm 230$ & $590 \pm 150$ & $-0.5 \%(0.2)$ & 0.004 & 0.49 \\
\hline Statins+niacin & $690 \pm 240$ & $590 \pm 140$ & $-0.7 \%(0.2)$ & 0.0002 & \\
\hline \multicolumn{6}{|l|}{ ICA lumen } \\
\hline Statins+placebo & $700 \pm 280$ & $700 \pm 270$ & $-0.2(0.9)$ & 0.81 & 0.98 \\
\hline Statins+niacin & $720 \pm 250$ & $720 \pm 250$ & $-0.2(0.9)$ & 0.84 & \\
\hline \multicolumn{6}{|l|}{ ICA lipid core } \\
\hline Statins+placebo & $47 \pm 36$ & $54 \pm 42$ & $0.2(0.4)$ & 0.66 & 0.13 \\
\hline Statins+niacin & $47 \pm 37$ & $33 \pm 24$ & $-0.8(0.5)$ & 0.10 & \\
\hline \multicolumn{6}{|l|}{ CC wall volume* } \\
\hline Statins+placebo & $110 \pm 40$ & $100 \pm 20$ & $-0.1 \%(0.2)$ & 0.52 & 0.26 \\
\hline Statins+niacin & $120 \pm 40$ & $100 \pm 20$ & $-0.4 \%(0.2)$ & 0.03 & \\
\hline \multicolumn{6}{|l|}{ CC lumen } \\
\hline Statins+placebo & $140 \pm 40$ & $150 \pm 40$ & $0.05(0.1)$ & 0.76 & 0.97 \\
\hline Statins+niacin & $160 \pm 40$ & $160 \pm 40$ & $0.04(0.2)$ & 0.80 & \\
\hline
\end{tabular}

the heterogeneity in combination therapies, lipid endpoints attained and endpoints measured, is worthwhile when comparing earlier trials, demonstrating a benefit of niacin, to the finding of no incremental benefit in the present study.

The HATS trial studied 160 patients with coronary disease and low HDL. ${ }^{9}$ Participants were assigned to a combination of simvastatin and niacin with or without antioxidants, or placebo. Three years of treatment with simvastatin and niacin resulted in greater regression of coronary angiographic atherosclerosis and fewer cardiovascular events compared with placebo.

The ARBITER-2 study enrolled secondary prevention patients with HDL less than $1.2 \mathrm{mmol} / \mathrm{L}$ (mean $1.0 \mathrm{mmol} / \mathrm{L}$ at baseline). ${ }^{8}$ All participants were on statins and were randomised to receive $1000 \mathrm{mg}$ niacin daily or placebo. Progression of carotid intimalmedial thickness (CIMT) occurred with statins alone, while no change was seen with niacin. However, between-group differences in CIMT were non-significant. The HDL and LDL Treatment Strategies in Atherosclerosis (ARBITER6-HALTS) trial enrolled secondary prevention patients with low HDL (mean $1.1 \mathrm{mmol} / \mathrm{L}$ ) who were randomised to statins in combination with niacin or ezetimibe, finding reduction in CIMT with statin and niacin therapy. ${ }^{23}$

The Oxford Niaspan study used MRI of the CCA to evaluate the impact of $2000 \mathrm{mg}$ nicotinic acid daily versus placebo in high-risk, statin-treated patients. ${ }^{10}$ Most $(65 \%)$ were diabetic. Niacin added to statins resulted in a significant decrease in CCA atherosclerosis.

This population is similar to prior niacin trials with regard to gender, cardiovascular risk factors and history of prior cardiovascular events. ${ }^{8-10} 23$ It is, however, unique in addressing participants of older age. Our study is notable for having the lowest attained LDL level for the on-treatment group of all these trials, at $1.7 \mathrm{mmol} / \mathrm{L}$ vs a range of $1.8-2.2 \mathrm{mmol} / \mathrm{L}$, as well as the highest attained HDL levels (1.5 vs $1.0-1.3 \mathrm{mmol} / \mathrm{L})$. Participants in the present study were able to attain these goals at a relatively modest mean statin dose $(20 \mathrm{mg} /$ day atorvastatin equivalent), in comparison with a mean dose of $33 \mathrm{mg} /$ day in Oxford and $40 \mathrm{mg} /$ day in HALTS. Neither HATS nor HALTS made direct comparison between statins alone and statins in combination with niacin. Differences in primary imaging outcome render direct comparison of outcomes, particularly with respect to magnitude and significance of change difficult to assess. HATS used luminal stenosis measured by quantitative coronary angiography, and therefore, was not capable of assessing the degree of externally remodelled atherosclerosis quantified by MR and ultrasound techniques. ARBITER-2 and HALTS used CIMT, which has been demonstrated to have excellent agreement with carotid MR imaging, albeit with significantly greater measurement variability. ${ }^{24}$ The Oxford Niaspan study used CCA MRI as the primary outcome, which may behave differently than the ICA with regard to atherosclerosis development and regression. ${ }^{25} 26$

Whether atherosclerosis regression in longitudinal imaging studies translates into a reduction in cardiovascular event rates is a subject of active debate. The finding of equivalent atherosclerosis regression with and without niacin here is mirrored in the failure to find a reduction in cardiovascular events with combination lipid-lowering therapy including niacin in the AIM-HIGH trial. ${ }^{12} 13$ This randomised controlled trial assigned 3414 participants to ER niacin at a dose the same or higher (1500$2000 \mathrm{mg} /$ day) used in the present study. Achieved on-treatment LDL in the active treatment arm $(1.7 \pm 0.6 \mathrm{mmol} / \mathrm{L})$, and the dose of statin required to reach that $(79 \%$ taking $40 \mathrm{mg}$ of simvastatin or less) were similar. The study entry and achieved HDL were significantly lower in AIM-HIGH $(0.9$ and $1.1 \mathrm{mmol} / \mathrm{L}$, respectively). The achieved relative difference in HDL between treatment arms was comparable between the two studies. The two studies also differ notably with regard to average age (older in the present study) and the prevalence of diabetes (34\% vs 19\%). Additionally, ezetimibe was permitted when necessary $(15.5 \%$ of patients) to achieve LDL targets in AIM-HIGH, while no additional lipid modifying agents were permitted in this study.

After 36 months of follow-up, AIM-HIGH demonstrated no difference in cardiovascular events between niacin-treated and placebo arms. This result has engendered much debate as to the reasons and appropriate interpretation of the negative outcome for niacin therapy, including questions of appropriate power, early termination, and concomitant therapy with ezetimibe and immediate release niacin. ${ }^{27}$ The still larger HPS2-THRIVE trial, ${ }^{12}$ which randomised 25673 patients to a maximum dose of $2000 \mathrm{mg}$ ER niacin/40 mg laropiprant, was subsequently terminated early with a failure to find benefit for additive niacin therapy, though results from this study await publication. The 
concordant results of the present study with larger events-driven studies may serve to shed light on the mechanism for the negative outcome in AIM-HIGH and THRIVE. Our findings suggest that the non-superiority of combined niacin and statin therapy relative to statin therapy alone result from comparable plaque regression with both therapeutic strategies, when patients are able to reach recommended LDL using statins. The present results are in keeping with findings in both primary and secondary prevention populations that the inverse association between HDL and cardiovascular risk is diminished or nullified at low levels of LDL. ${ }^{28-30}$

\section{Limitations}

The results of this study are pertinent to statin-treated individuals at high cardiovascular risk who are able to attain recommended LDL levels on statin therapy. Participants in this study did not have marked elevations in overall lipoprotein load at entry, and these results may not be generalisable to such patients. While there were statistically and clinically significant differences in HDL at 18 months, the 5\% increase from baseline in the niacin arm was modest. This occurred despite $88 \%$ of the niacin arm reaching their protocol-specified target dose of $1500 \mathrm{mg}$ daily. The HDL level of placebo-treated participants in this study was comparable with the active treatment arms of similar niacin trials. Although the follow-up in this study was longer than any previously reported imaging studies of niacin and carotid atherosclerosis, we cannot exclude that effects of niacin on carotid wall volume may have a longer time course than was examined here. Similarly, we cannot exclude the possibility that niacin has effects on plaque regression of a magnitude smaller than this study was powered to detect.

\section{CONCLUSIONS}

Treatment of older individuals with documented atherosclerosis with statin therapy to low LDL, with or without niacin, produced significant reduction in carotid atherosclerosis. The addition of niacin in patients already well treated with statins did not result in greater plaque regression.

\section{Acknowledgements We extend our thanks to Margene Kennedy CRNP and Rosalie Cosgriff RN for their expert patient care.}

Contributors CTS and JACL are responsible for the overall content as guarantors. ALV, IG, CC, MM, AS, GG, VF, BAW and DAB participated in the study design, data collection and review and edits to the final manuscript.

Funding This work was supported by grant AG-021570 from the National Institute on Aging. Kos Pharmaceuticals, later acquired by Abbott Pharmaceuticals provided study drug at no cost and funding to complete data analysis. Dr Sibley was supported by grant T32-HL-007227-35 and by the intramural research program of the NIH Clinical Center. Funding sources had no role in study design and conduct; data collection, interpretation or analysis; or in manuscript preparation or approval.

\section{Competing interests None.}

Patient consent Obtained.

Ethics approval The findings of this report are those of the authors, and do not reflect views of the National Institute on Aging or the National Institutes of Health.

Provenance and peer review Not commissioned; externally peer reviewed.

\section{REFERENCES}

1 Lloyd-Jones D, Adams RJ, Brown TM, et al. Heart disease and stroke statistics2010 update: a report from the American Heart Association. Circulation 2010; 121:e46-215.

2 Education Program (NCEP) Expert Panel on Detection, Evaluation, and Treatment of High Blood Cholesterol in Adults (Adult Treatment Panel III). JAMA 2001;285:2486-97.

3 Gordon DJ, Probstfield JL, Garrison RJ, et al. High-density lipoprotein cholesterol and cardiovascular disease. Four prospective American studies. Circulation 1989:79:8-15.
4 Prospective Studies Collaboration. Blood cholesterol and vascular mortality by age sex, and blood pressure: a meta-analysis of individual data from 61 prospective studies with 55000 vascular deaths. The Lancet 2007;370:1829-39.

5 Voight BF, Peloso GM, Orho-Melander M, et al. Plasma HDL cholesterol and risk of myocardial infarction: a mendelian randomisation study. The Lancet 2012;380:572-80.

6 Azen SP, Mack WJ, Cashin-Hemphill L, et al. Progression of coronary artery disease predicts clinical coronary events: long-term follow-up from the cholesterol lowering atherosclerosis study. Circulation 1996;93:34-41.

7 Canner PL, Berge KG, Wenger NK. Fifteen year mortality in Coronary Drug Project patients: long-term benefit with niacin. J Am Coll Cardiol 1986;8:1245-55.

8 Taylor AJ, Sullenberger LE, Lee HJ, et al. Arterial Biology for the Investigation of the Treatment Effects of Reducing cholesterol (ARBITER) 2: a double-blind, placebocontrolled study of extended-release niacin on atherosclerosis progression in secondary prevention patients treated with statins. Circulation 2004;110:3512-17.

9 Brown BG, Zhao X-Q, Chait A, et al. Simvastatin and niacin, antioxidant vitamins, or the combination for the prevention of coronary disease. NEJM 2001;345:1583-92.

10 Lee JMS, Robson MD, Yu L-M, et al. Effects of high-dose modified-release nicotinic acid on atherosclerosis and vascular function: a randomized, placebo-controlled, magnetic resonance imaging study. J Am Coll Cardiol 2009;54:1787-94.

11 Taylor AJ, Lee HJ, Sullenberger LE. The effect of 24 months of combination statin and extended-release niacin on carotid intimamedia thickness: ARBITER 3. Curr Med Res Opin 2006;22:2243-50.

12 HPS2-THRIVE Collaborative Group. HPS2-THRIVE randomized placebo-controlled trial in 25673 high-risk patients of ER niacin/laropiprant: trial design, pre-specified muscle and liver outcomes, and reasons for stopping study treatment. Eur Heart 2013;34:1279-91.

13 The AIM-HIGH Investigators. Niacin in patients with low HDL cholesterol levels receiving intensive statin therapy. N Engl J Med 2011;365:2255-67.

14 Heart Protection Study Group. MRC/BHF Heart Protection Study of cholesterol lowering with simvastatin in 20,536 high-risk individuals: a randomised placebo controlled trial. The Lancet 2002;360:7-22.

15 Zulman D, Sussman J, Chen $X$, et al. Examining the evidence: a systematic review of the inclusion and analysis of older adults in randomized controlled trials. J Gen Intern Med 2011:26:783-90.

16 Grundy SM, Becker D, Clark LT, et al. Detection, evaluation, and treatment of high blood cholesterol in adults (Adult Treatment Panel III). Circulation 2002;106:3143-421.

17 Wasserman BA, Smith WI, Trout lii HH, et al. Carotid artery atherosclerosis: in vivo morphologic characterization with gadolinium-enhanced double-oblique MR imaging - initial results. Radiology 2002;223:566-73.

18 Wasserman BA, Sharrett AR, Lai $S$, et al. Risk factor associations with the presence of a lipid core in carotid plaque of asymptomatic individuals using high-resolution MRI: the Multi-Ethnic Study of Atherosclerosis (MESA). Stroke 2008;39:329-35.

19 Adame IM, Van Der Geest RJ, Wasserman BA, et al. Automatic segmentation and plaque characterization in atherosclerotic carotid artery MR images. Magn Reson Materi Phys Biol Med 2004;16:227-34.

20 Lima JA, Desai MY, Steen $\mathrm{H}$, et al. Statin-induced cholesterol lowering and plaque regression after 6 months of magnetic resonance imaging-monitored therapy. Circulation 2004;110:2336-41.

21 Corti R, Fayad ZA, Fuster V, et al. Effects of lipid-lowering by simvastatin on human atherosclerotic lesions: a longitudinal study by high-resolution, noninvasive magnetic resonance imaging. Circulation 2001;104:249-52.

22 Michos ED, Sibley CT, Baer JT, et al. Niacin and statin combination therapy for atherosclerosis regression and prevention of cardiovascular disease events: reconciling the AIM-HIGH (atherothrombosis intervention in metabolic syndrome with low HDL/high triglycerides: impact on global health outcomes) trial with previous surrogate endpoint trials. J Am Coll Cardiol 2012;59:2058-64.

23 Taylor AJ, Villines TC, Stanek EJ, et al. Extended-release niacin or ezetimibe and carotid intima-media thickness. NEJM 2009;361:2113-22.

24 Duivenvoorden $\mathrm{R}$, de Groot $\mathrm{E}$, Elsen $\mathrm{BM}$, et al. In vivo quantification of carotid artery wall dimensions: 3.0-Tesla MRI versus B-mode ultrasound imaging. Circulation Cardiovasc Imaging 2009;2:235-42.

25 Stein JH, Douglas PS, Srinivasan SR, et al. Distribution and cross-sectional age-related increases of carotid artery intima-media thickness in young adults: The Bogalusa Heart Study. Stroke 2004;35:2782-87.

26 Polak JF, Pencina MJ, Pencina KM, et al. Carotid-wall intima-media thickness and cardiovascular events. N Engl J Med 2011;365:213-21.

27 Nicholls SJ. The AIM-HIGH (Atherothrombosis Intervention in Metabolic Syndrome with low HDL/High Triglycerides: Impact on Global Health Outcomes) Trial: To Believe or Not to Believe? J Am Coll Cardiol 2012;59:2065-67.

28 Ray KK, Cannon CP, Cairns R, et al. Prognostic utility of ApoB/Al, total cholesterol/ $\mathrm{HDL}$, non-HDL cholesterol, or hs-CRP as predictors of clinical risk in patients receiving statin therapy after acute coronary syndromes: results from PROVE IT-TIMI 22. Arterioscler Thromb Vasc Biol 2009;29:424-30.

29 Barter P, Gotto AM, LaRosa JC, et al. HDL cholesterol, very low levels of LDL cholesterol, and cardiovascular events. NEJM 2007;357:1301-10.

30 Ridker PM, Genest J, Boekholdt SM, et al. HDL cholesterol and residual risk of first cardiovascular events after treatment with potent statin therapy: an analysis from the JUPITER trial. Lancet 2010;376:333-39. 